\title{
A VLBI study of dying and restarting radio sources
}

\author{
P. Parma, F. Mantovani, H. R. de Ruiter, K.-H. Mack \\ INAF-Istituto di Radioastronomia \\ Bologna, Italy \\ E-mail: parmaeira.inaf.it
}

\section{Murgia, F. Govoni}

INAF-Osservatorio Astronomico di Cagliari

Cagliari, Italy

We discuss some radio sources in which the energy supply from the nucleus has been switched off, as can be deduced from the overall steep radio spectrum. Such sources are aptly called dying, although it may also happen that new activity may occur in the nucleus, while the large scale structure was produced in a previous cycle. A restarting radio source may therefore show two overlapping phases of activity. We selected a number of dying or restarting radio sources for further study with the European VLBI Network (EVN) and MERLIN.

Here we present EVN and MERLIN observations of four radio sources; of those two are probable restarting sources, while the other two are CSS, which are likely to be in a phase of inactivity and therefore dying.

In the EVN and MERLIN images of the two restarting sources only the radio cores are detected and their strength is consistent with the VLA flux densities found at higher frequencies. In both cases the extended structure has been resolved out completely.

The two compact steep spectrum sources show extended structure in the MERLIN images; in the former MERLIN also detects a very faint core, whereas the EVN image shows just the faint core and nothing else. In the latter only the southern component is detected by the EVN, and is resolved in two faint and compact components.

The structures found in the two CSS suggest that these sources are indeed dying: the flux remaining in the radio cores is very weak compared to the total extended flux (which is resolved out in the EVN observations), and, although not completely switched off, the cores are probably fading gradually.

$10^{\text {th }}$ European VLBI Network Symposium and EVN Users Meeting: VLBI and the new generation of radio arrays

Manchester, $U K$

September 20-24, 2010 


\section{Introduction}

In some sources the nuclear activity has been switched off, so that no refueling of new energetic electrons to the radio lobes takes place. As a result of the ageing of the lobes the radio spectra become ever steeper.

Detailed spectral ageing models for fading radio galaxies have been developed by Komissarov \& Gubanov ([1]) and Slee et al ([5]). These models assume a finite duration for the continuous injection of relativistic electrons, followed by a relic phase. In both phases the electrons lose energy by synchrotron emission and inverse Compton scattering of the cosmic microwave background photons. Extremely steep integrated spectra are consistent with a scenario in which the injection of fresh electrons has ceased for a significant fraction of the total source lifetime.

As this dying phase has a relatively short duration it is expected that such sources are rare. Indeed, selection of candidates has been done using surveys of many thousands of radio sources. Only by cross correlating, for example, the WENSS and NVSS data (at respectively $325 \mathrm{MHz}$ and $1.4 \mathrm{GHz}$ ) a few dozen dying sources are found (Murgia et al. [2]; Parma et al. 2007 [3]).

\section{The EVN and MERLIN observations}

Parma et al. ([3]) defined a sample of six dying and three restarting sources based on WENSS, NVSS and high resolution VLA data. Four sources were selected for further study, two restarting sources WNB $1328.3+6520$ and WNB 1438.9+3720, and two possible dying Compact Steep Spectrum sources (CSS), WNB 1408.8+4630 and WNB 1458.0+4959.

In Table 1 we give some information on the EVN observations. These were done on November 9, 2009, using the following telescopes: Ef, Nt, Tr, Jb, Da, On, Mc, Ur, Sh, Wb. The observing frequency was $1658 \mathrm{MHz}$. Note that WNB 1458.0+4959 has two detected VLBI components (A being the eastern, and B the western component).

Table 1: EVN observations

\begin{tabular}{lllllllll}
\hline Source & flux & rms & size & size & P.A. & beam & beam & P.A. \\
& $\mathrm{S}_{\text {tot }}$ & $3 \sigma$ & major & minor & & $\begin{array}{l}\text { major } \\
\text { minor }\end{array}$ & \\
& mJy & mJy & mas & mas & deg. & mas & mas & deg. \\
WNB 1328.3+6520 & 1.4 & 0.10 & 6.8 & - & 173 & 11.4 & 7.9 & -60 \\
WNB 1408.8+4630 & 5.8 & 0.10 & 7.9 & 3.9 & 85 & 10.8 & 8.8 & -52 \\
WNB 1438.9+3720 & 1.46 & 0.10 & - & - & - & 11.4 & 8.0 & -83 \\
WNB 1458.0+4959 A & 0.60 & 0.05 & 2.0 & - & 34 & 12.2 & 10.9 & 70 \\
WNB 1458.0+4959 B & 0.97 & & & & & & & \\
\hline
\end{tabular}

In Table 2 we summarize the MERLIN observations, which were done simultaneously with the EVN observations, at $1.6 \mathrm{GHz}$. 
Table 2: MERLIN observations

\begin{tabular}{lllllllll}
\hline Source & flux & rms & size & Size & P.A. & beam & beam & P.A. \\
& $\mathrm{S}_{\text {tot }}$ & $3 \sigma$ & major & minor & & major & minor & \\
& mJy & mJy & arcs & arcs & deg. & arcs & arcs & deg. \\
WNB 1328.3+6520 & 3.00 & 0.27 & 0.15 & 0.11 & 154 & 0.22 & 0.17 & -20 \\
WNB 1408.8+4630 & 7.20 & 0.33 & 0.07 & 0.02 & 159 & 0.39 & 0.15 & -34 \\
WNB 1438.9+3720 & 1.35 & 0.27 & 0.65 & 0.16 & 178 & 0.71 & 0.52 & -17 \\
WNB 1458.0+4959 & 4.10 & 0.24 & 2.00 & 1.20 & 130 & 0.58 & 0.58 & -2 \\
\hline
\end{tabular}

\section{Results}

In Figs. 1-4 we present the MERLIN and EVN images for the two CSS sources WNB $1328.3+6520$ and WNB1458.0+4959.

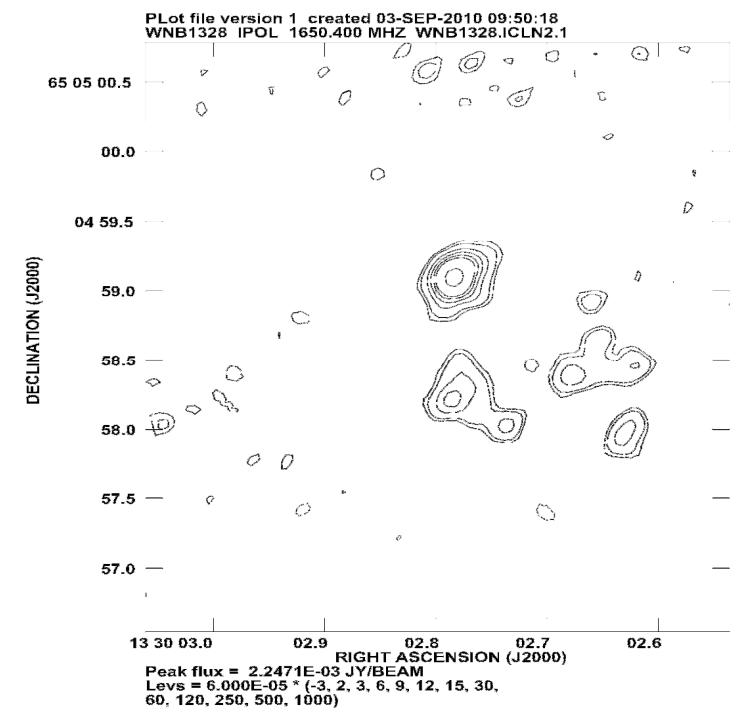

Fig. 1: WNB1328.3+6520, MERLIN image

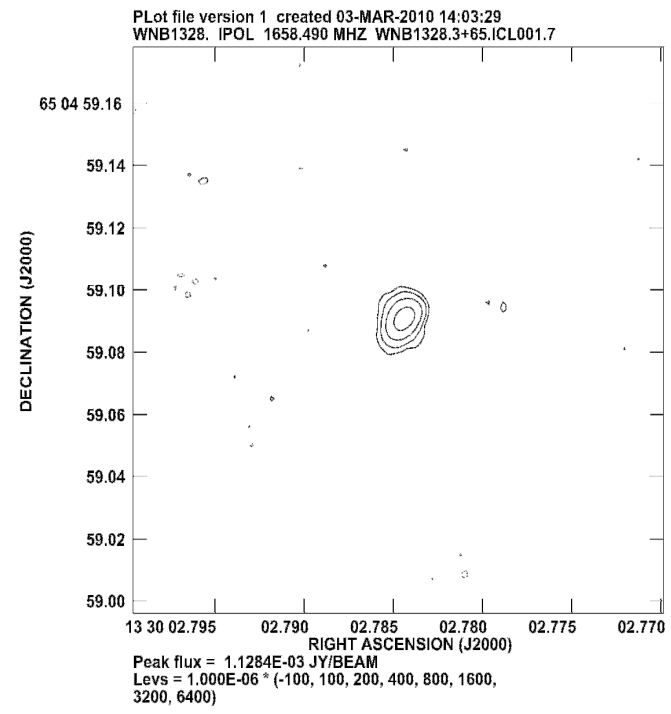

Fig. 2: WNB1328.3+6520, EVN image

In both the MERLIN and EVN images of the two restarting sources (WNB 1408.8+4630 and WNB $1438.9+3620$ ) the radio cores are detected, and the strength of the cores is consistent with the VLA core flux densities found at higher frequencies. In both cases the extended structure has been resolved out completely.

In the CSS WNB 1328.3+6520 MERLIN detects a very faint core and some extended structure, but the EVN observations show just the very faint core. WNB 1458.0+4959 has multiple structure in both the MERLIN and EVN images: the EVN data only show the southern MERLIN component, resolved into two faint and compact components. Possibly WNB 1458.0+4959 is a switched-off CSO (see Readhead et al. [4]). 


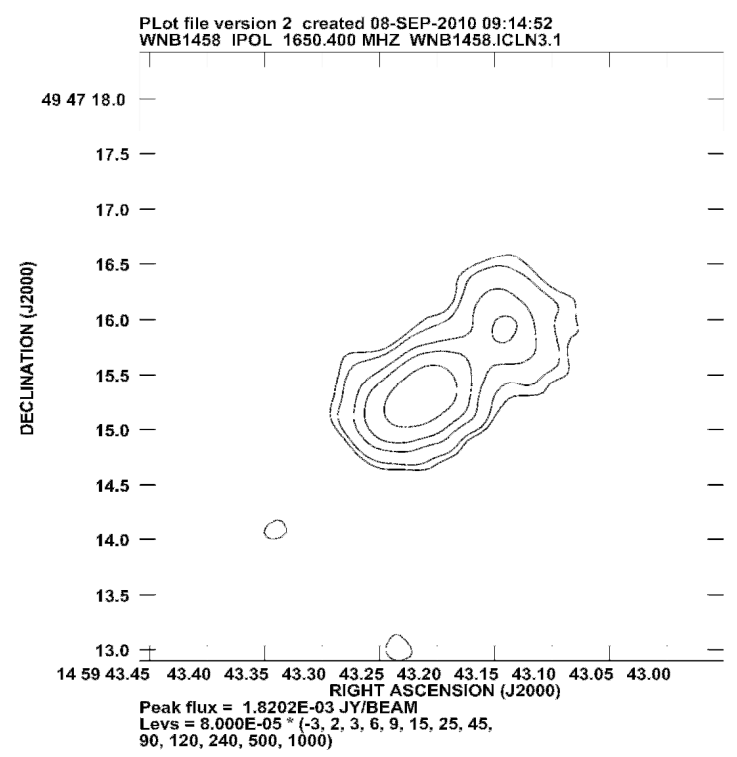

Fig. 3: WNB1458.0+4959, MERLIN image

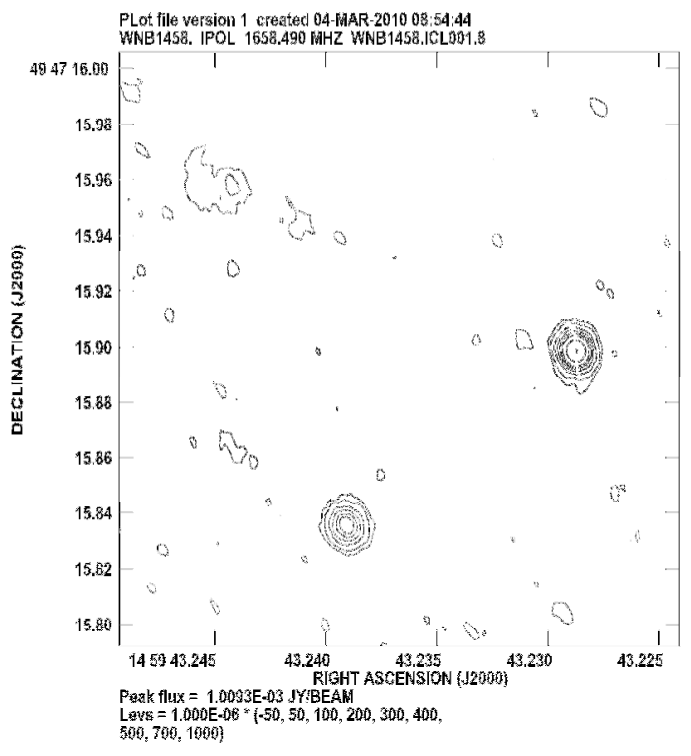

Fig. 4:WNB 1458.0+4959, EVN image

The structures in the two possible CSSs suggest that they are indeed dying: the flux remaining in the radio cores is very weak compared to the total extended flux, that has been mostly resolved out in the MERLIN observations and completely in the VLBI data. In both cases the core has not been switched off entirely, but is probably fading gradually; it is anyway so weak that it does not add significantly to the integrated spectra. This is particularly clear in WNB 1458.0+4959, in which the extended structure visible in the Merlin image is entirely absent in the EVN observations, and only two very weak compact components (one of which presumably the radio core) are left that hardly contribute to the overall flux density.

\section{References}

[1] S.S. Komissarov and A.G. Gubanov, 1994, Relic radio galaxies: evolution of the synchrotron spectrum, A\&A, 285, 27

[2] M. Murgia, P. Parma, K.-H. Mack, et al., 2010, Dying radio galaxies in clusters, A\&A, in press

[3] P. Parma, M. Murgia, H.R. de Ruiter, R. Fanti, K.-H. Mack and F. Govoni, 2007, In search of dying radio sources in the local universe, A\&A, 470, 875

[4] A.C.S. Readhead, W. Xu, T.J. Pearson, P.N. Wilkinson and A.G. Polatidis, 1994, Compact Symmetric objects, in Compact extragalactic radio sources, Proceedings of the NRAO workshop held at Socorro, eds. J.A. Zensus and K. Kellerman, p. 17

[5] O.B. Slee, A.L. Roy, M. Murgia, H. Andernach, \& M. Ehle, 2001, Four extreme relic radio sources in clusters of galaxies, AJ, 122, 1172 